\title{
FORO
}

Recibido 14.12.2012. Aprobado 21.08.2013

Evaluado por el sistema double blind review. Editor Científico: Mário Aquino Alves, Ezequiel Reficco y Juan Arroyo

DOI: http://dx.doi.org/10.1590/So034-759020140104

\section{CREDIBILIDAD EN LATINOAMÉRICA DEL INFORME DE RESPONSABILIDAD SOCIAL CORPORATIVA}

\author{
Credibilidade do relatório de responsabilidade social corporativa na \\ América Latina
}

Credibility in Latin America of corporate social responsibility reports

\section{LAURA SIERRA-GARCÍA \\ Insiegar@upo.es \\ Profesora de la Facultad de \\ Empresariales, Universidad Pablo de \\ Olavide, Sevilla - España}

\section{MARÍA A. GARCÍA-BENAU}

maria.garcia-benau@uv.es Profesora de la Facultad de Economía, Universidad de Valencia, Valencia - España

\section{ANA ZORIO}

ana.zorio@uv.es

Profesora de la Facultad de Economía, Universidad de Valencia, Valencia - España

\begin{abstract}
RESUMEN
Para dar mayor credibilidad a la información publicada en los informes de responsabilidad social corporativa se asigna a estos de un proceso de aseguramiento por parte de un experto externo. Esta investigación se basa en 1.021 observaciones de 12 países de Latinoamérica durante los años 20062010. A la vista de los resultados, podemos concluir que Latinoamérica es una región donde cada vez hay un mayor compromiso con la sostenibilidad. Además, los resultados señalan que la decisión de otorgar mayor credibilidad a las políticas sostenibles por medio del aseguramiento depende del tamaño de la empresa, del sector al que pertenece y del país donde se encuentre.
\end{abstract}

PALABRAS CLAVES / Responsabilidad social corporativa, aseguramiento, auditores, latinoamérica, GRI.

\section{RESUMO}

Para dar mais credibilidade às informações publicadas nos relatórios de responsabilidade social corporativa, um processo de garantia é atribuído a estes por um perito externo. Esta pesquisa baseiase em 1.021 observações de 12 países da América Latina durante os anos 2006-2010. Diante dos resultados, conclui-se que a América Latina é uma região com um compromisso cada vez maior com a sustentabilidade. Além disso, os resultados indicam que a decisão de dar mais credibilidade às políticas sustentáveis por meio da garantia depende do tamanho da empresa, do setor ao qual ela pertence e do país onde ela está.

PALAVRAS-CHAVE | Responsabilidade social corporativa, garantia, auditores, América Latina, GRI.

\section{ABSTRACT}

To improve credibility of Corporate Social Responsibility reports, they are assured by an external expert. This research is based on 1,021 observations from 12 countries in Latin America during the years 2006-2010. In view of the results obtained, we can conclude that Latin America is a region where there is a great commitment to sustainability. In addition, our results indicate that the decision to give more credibility to sustainable policies through the assurance process depends on the size of the RSC reporting company, the industry to which it belongs and the country where the firm operates.

KEY WORDS / Corporate social responsibility, assurance, auditors, Latin America, GRI. 


\section{INTRODUCCIÓN}

Hoy en día sería difícil pensar en las empresas como entidades orientadas únicamente a la generación de beneficios para los socios. La creación de valor social ha adquirido, poco a poco, un puesto relevante junto a la creación de valor económico.

Aunque ya se detectaron prácticas empresariales socialmente responsables en la segunda mitad del siglo XX (Carroll, 1999), es a principio del siglo XXI cuando se ha dado un verdadero impulso a lo que viene denominándose Responsabilidad Social Corporativa (RSC). Reficco (2010) habla de la relación entre RSC y los negocios inclusivos, matizando que la primera no incluye a la segunda (También puede consultarse Reficco, 2009).

América Latina constituye un caso de estudio muy interesante, ya que empezó muy temprano a ser consciente de la necesidad de incrementar el compromiso social de las empresas. Los acontecimientos acaecidos en la década de los 80 fueron especialmente relevantes debido al mayor protagonismo adquirido por el sector privado y a las limitaciones de las políticas públicas, lo que llevó a que las cuestiones medioambientales y sociales entraran a formar parte de las agendas corporativas (Véase Reficco \& Ogliastri, 2009 o Gómez Villegas, 2008 para un estudio del caso en una empresa cementera colombiana). De hecho, pueden encontrarse iniciativas a nivel local del ámbito latinoamericano sobre directrices y recomendaciones de RSC. En este sentido, Berbel Giménez, Reyes Gómez, \& Gómez Villegas (2007) presentan una interesante clasificación de las mismas (que incluyen, por ejemplo, el Índice Colombiano de Responsabilidad Empresarial o los indicadores Ethos, de Brasil).

La calidad de la información contenida en los informes de sostenibilidad se alza como un requisito cada vez más exigido por los grupos de interés. Por ello, muchas empresas aseguran sus informes de RSC como una forma de garantizar la credibilidad de la información publicada.

El objetivo de este artículo es analizar las tendencias en la presentación del informe de aseguramiento RSC por parte de las empresas latinoamericanas y las características corporativas que determinan distintas estrategias empresariales en este campo. Para ello, realizamos un estudio empírico a partir de una muestra de 1.021 observaciones de 12 países de Latinoamérica durante el período comprendido entre 2006 y 2010, utilizando la base de datos del Global Reporting Initiative (GRI). Nuestro estudio empírico se centra en los informes de verificación de las memorias de sostenibilidad. Nuestro análisis presenta los resultados obtenidos en dos etapas. En una primera etapa, examinamos la situación de verificación de los informes de sostenibilidad a partir de la presentación de resultados de corte descriptivo y, en una segunda etapa, analizamos de forma exploratoria las características corporativas que explican que las empresas verifiquen o no sus informes de sostenibilidad.

Cabe señalar que esta investigación presenta varios aspectos a destacar, como son su carácter pionero ya que el tema del aseguramiento de informes de RSC es un tema aún no muy explorado, la solidez que se deriva de haber utilizado una muestra suficientemente amplia y su oportunidad, pues los datos utilizados son recientes y es un tema de actualidad en el marco regulador y en el ámbito de la organización y dirección de empresas.

La estructura de este artículo es la siguiente. Tras esta breve introducción, en la que se destaca la oportunidad e interés de esta investigación, el apartado segundo ofrece una revisión de la literatura previa sobre el tema objeto de estudio, esto es, RSC y aseguramiento. En el tercer apartado, se presenta la metodología y la muestra empleada en el estudio empírico realizado. El cuarto epígrafe presenta una visión descriptiva de la situación de verificación de los informes de sostenibilidad y los resultados de un estudio exploratorio sobre los factores que conducen a la decisión de verificar. Por último se muestran las conclusiones alcanzadas.

\section{RESPONSABILIDAD SOCIAL CORPORATIVA Y ASEGURAMIENTO}

Aunque la tendencia por fortalecer el compromiso de la empresa con sus grupos de interés se inició entre las grandes compañías multinacionales (Kolk, 2008), cada vez son más las empresas que publican informes de Responsabilidad Social Corporativa (Kpmg, 2011). El principal objetivo de las memorias de sostenibilidad gira en torno a la información sobre el desempeño organizativo en los términos de su contribución/detracción a la sostenibilidad (Gray \& Milne, 2004, Porter \& Kramer, 2002; Austin, Gutiérrez, Ogliastri, \& Reficco , 2009; Giannarakis \& Theotokas, 2011). Según los resultados de una encuesta llevada a cabo por KMPG en noviembre de 2011 a 350 directores financieros de organizaciones en todo el mundo, la sostenibilidad puede servir para fortalecer la competencia, incrementar la innovación y mejorar la reputación de las empresas (Kpmg, 2011).

Fernandez \& Quiota (2011) en un estudio realizado con empresas brasileñas en el período 2001-2007 concluyen que existe una correlación positiva entre la inversión socio ambiental y el desempeño financiero (véase también Aragón-Correa, Hurtado-Torres, Sharma, \& García-Morales, 2008).

Feldman \& Reficco (2012) analizaron y encontraron evidencia empírica, por medio de un diseño experimental, de la 
incidencia que la RSC tiene en el comportamiento de compra y en la disposición a pagar por atributos de este tipo en las decisiones de compra de los consumidores. Arroyo (2012) en su estudio compara los comportamientos seguidos en Europa y en América Latina respecto a la RSC concluyendo que los países europeos se encuentran más comprometidos con la RSC que los países Latinoamericanos, aunque existen diferencias importantes entre los distintos países de ambas áreas geográficas.

La literatura sobre desempeño también ha estudiado el efecto de la elaboración de informes sobre RSC (véase entre otros Orlitzky, Schmidt, \& Rynes, 2003; ARAGÓN-CORREA, Hurtado-Torres, Sharma, \& García-Morales, 2008; KangLee, \& Huh, 2010; García-Benau, Sierra, \& Zorio, 2013). Siguiendo a Kang Lee, \& Huh (2010) podemos clasificarlos en tres grupos: un escaso número de trabajos que encuentran una relación negativa (por ejemplo, Wright \& Ferris, 1997), un segundo grupo que encuentra una relación positiva (Orlitzky, Schmidt, \& Rynes, 2003; ARAGÓN-CORREA y otros, 2008; Simpson \& Kohers, 2002; García-Benau,Sierra, \& Zorio, 2013) y por último, otros que no encuentran relación u obtienen resultados mixtos (Nelling \& Webb, 2009; Kang, Lee, \& Huh, 2010).

La mayoría de los informes de sostenibilidad se elaboran siguiendo las recomendaciones establecidas en el Global Reporting Initiative (GRI). Según datos de KPMG (2011) el 80\% de las compañías del Global Fortune 250 siguen el GRI para elaborar su informe de RSC. Los niveles de cumplimiento de las guías GRI abarcan las tres dimensiones de la RSC (económica, social y medioambiental), expresando el nivel de cumplimiento con las letras A, B y C (A corresponde al nivel de aplicación más elevado, B al intermedio y C al más bajo).

La presentación de informes de sostenibilidad y el afán por incrementar la calidad de la información divulgada ha dado un impulso importante a la verificación de estos informes por medio de los denominados informes de aseguramiento (o verificación). Sin embargo, no existe ninguna legislación de tipo obligatorio que regule los encargos de verificación, si bien en fechas recientes se han publicado algunos marcos teóricos que ayudan a su desarrollo (laasb, 2003; Fee, 2006; Accountability, 2003 y 2008).

Las empresas están llevando a cabo el aseguramiento de sus informes de RSC como una forma de transparencia empresarial. El GRI incorpora el signo + a sus tres niveles de cumplimiento establecidos ( , B y C) para indicar que el informe de RSC ha sido sometido a verificación externa.

De acuerdo con los datos publicados en el Informe de Sostenibilidad en la UE (2010), el 49\% de las memorias de RSC publicadas en 2008 realizaron algún tipo de aseguramiento y el $68 \%$ aplicaron el estándar del GRI. Los datos del GRI de 2010 co- rroboran esta tendencia pues casi la mitad de los informes han sido asegurados, lo que supone un aumento con respecto a los datos de 2009 (del 45\% al 47\%) (Puede consultarse Fonseca, 2010 para un análisis crítico entre las empresas mineras). Moroney, Windsor, \& AW, (2012) concluyen que el aseguramiento externo del informe de sostenibilidad mejora su calidad, aunque también hay estudios en la literatura que señalan algunas debilidades existentes en el propio proceso de aseguramiento (O’dwyer, 2011).

Sin embargo, en la literatura internacional son pocos los trabajos que abordan la práctica de verificación de los informes de sostenibilidad y su papel en la mejora de la credibilidad de la información (Knechel, Wallage, Eilifsen, \& Praag, 2006; Adams \& Evans, 2004; Simnett, Vanstraelen, \& Chua, 2009; Fifka, 2013). Algunos trabajos están centrados en el análisis de quiénes son los expertos que llevan a cabo el informe de aseguramiento, estudiando el papel de los auditores y de los consultores (Véase Hasan, Maijoor, Mock, Roebuck, Simnett, \& Vanstraelen , 2005; Asif, Searcy, Dos Santos, \& Kensah, 2012).

A la vista de los hallazgos de la literatura previa, en el marco latinoamericano nos planteamos testar las siguientes preguntas de investigación: Q1: ¿Hay diferencias entre las empresas que aseguran ver-
sus las que no aseguran el informe de RSC con respecto a
las variables año, tamaño, sector sensible y país?

Q2: ¿El aseguramiento de las memorias de RSC depende de algunas variables corporativas, tales como el año, el tamaño y el sector sensible al que pertenece la empresa y la pertenencia a uno de los países?

\section{MUESTRA Y METODOLOGÍA}

El ya comentado Global Reporting Initiative (GRI) es una institución independiente que creó el primer estándar mundial de elaboración de informes de RSC o memorias de sostenibilidad. Para la realización de nuestro estudio empírico nos hemos basado en la información sobre las empresas que elaboran dicha institución sobre dichas memorias.

Nuestra investigación se centra en las empresas Latinoamericanas y analiza la tendencia seguida por dichas empresas. El período de estudio abarca desde del 2006, primer año del que existe información acerca del aseguramiento del informe de RSC, hasta el año 2010, último disponible en la fecha de recopilación de datos. La muestra comprende un total de 1.021 observaciones localizadas en 12 países de la región de Latinoamérica. 
En esta investigación hemos llevado a cabo el siguiente trabajo. En primer lugar, hemos estudiado la tendencia de las empresas latinoamericanas en asegurar el informe de RSC, en función de una serie de características corporativas y del año. Para ello hemos realizado un análisis por medio de tablas de contingencias univariante. En segundo lugar, se presenta un estudio empírico con un modelo de regresión logística que nos explica de qué factores depende que las empresas aseguren su informe de RSC. Al utilizar como variable dependiente si la empresa asegura su informe de RSC o no (variable dicotómica) y como variable dependientes variables categóricas la técnica más apropiada dentro del análisis multivariante es la regresión logística y así lo demuestra la literatura existente (Véase, Simnett, Vanstraelen, \& Chua, 2009; Kolk \& Perego, 2010; Añadir Zorio, García-Benau, \& Sierra, 2013). El modelo de regresión logístico lo hemos definido como sigue:

Aseguramiento $_{\text {it }}=\beta_{1}$ año $+\beta_{2}$ tamaño $+\beta_{3}$ sector sensible $+\beta_{4}$ pais $+\varepsilon_{\text {it }}$

La variable dependiente aseguramiento de las memorias de RSC es una variable dicotómica que toma el valor 1 si las empresas aseguran el informe de RSC y o en caso contrario. Las variables independiente son el año (variable categórica desde el año 2006 hasta 2010), el tamaño (variable dicotómica donde toma el valor 1 si la empresa es pequeña 0 mediana y o en caso contrario), el sector sensible al que pertenece la empresa (variable dicotómica donde toma el valor 1 si la empresa pertenece al sector sensible y o en caso contrario) y la pertenencia a uno de los países de los que se componen la muestra (variable con 12 categorías por cada uno de los países de Latinoamérica).

\section{RESULTADOS DEL ESTUDIO EMPÍRICO DEL ASEGURAMIENTO DE LOS INFORMES DE RSC}

En este apartado describimos, por un lado, la muestra objeto de estudio, y por otro, los resultados del análisis empírico realizado a empresas latinoamericanas desde el año 2006 al año 2010.

\section{Resultados de tipo descriptivo}

La tabla 1 muestra el detalle de las observaciones que presentan el informe de RSC en Latinoamérica por años (nótese que en algunos países latinoamericanos ninguna empresa lo presenta según el GRI, siendo solo 12 los países incluidos). Como se puede ver, en el ámbito latinoamericano existe una tendencia al alza de la presentación de estas memorias al GRI, pasando, en el año 2006, de 80 empresas a 342 empresas, en el año 2010. Ello supone haber cuadriplicado el total de empresas que presentan su informe de RSC en 5 años.

\section{TABLA 1. Informes de sostenibilidad presentado de empresas latinoamericanas entre 2006- 2010}

\begin{tabular}{l|l|l|l|l|l|l}
\hline \multirow{2}{*}{} & \multicolumn{2}{|l}{ AÑO DEL INFORME DE RESPONSABILIDAD } & \multirow{2}{*}{ Total } \\
\cline { 2 - 6 } & 2006 & 2007 & $\mathbf{2 0 0 8}$ & $\mathbf{2 0 0 9}$ & $\mathbf{2 0 1 0}$ & \\
\hline Total & 80 & 142 & 191 & 266 & 342 & 1.021 \\
\hline
\end{tabular}

Si nos centramos en el aseguramiento de estos informes, podemos observar, tal y como muestra la tabla 2 que, del total de las empresas que informan al organismo, el $28 \%$ aseguran sus informes de RSC. El total de empresas que lo aseguran aumenta de 24 en 2006 a 99 en 2010 (que de nuevo supone cuadriplicar el número de empresas que lo aseguran y por ello oscilan poco los porcentajes de empresas que aseguran sus informes de RSC con respecto al total de empresas que elaboran informes de RSC).

\section{TABLA 2. Empresas que aseguran vs que no aseguran el informe de RSC}

\begin{tabular}{|c|c|c|c|c|c|c|c|c|c|c|c|}
\hline \multirow[b]{3}{*}{ ASEGURAN } & \multicolumn{10}{|c|}{ AÑO DEL INFORME DE RESPONSABILIDAD } & \multirow{3}{*}{$\begin{array}{c}\text { Total } \\
289\end{array}$} \\
\hline & \multicolumn{2}{|c|}{2006} & \multicolumn{2}{|c|}{2007} & \multicolumn{2}{|c|}{2008} & \multicolumn{2}{|c|}{2009} & \multicolumn{2}{|c|}{2010} & \\
\hline & 24 & $30 \%$ & 37 & $26 \%$ & 54 & $28 \%$ & 75 & $28 \%$ & 99 & $29 \%$ & \\
\hline NO ASEGURAN & 56 & $70 \%$ & 105 & $74 \%$ & 137 & $72 \%$ & 191 & $72 \%$ & 243 & $71 \%$ & 732 \\
\hline Total & 80 & $100 \%$ & 142 & $100 \%$ & 191 & $100 \%$ & 266 & $100 \%$ & 342 & $100 \%$ & 1.021 \\
\hline
\end{tabular}


Cuando analizamos la muestra por países (ver tabla 3) observamos un aspecto que resulta de suma relevancia. Brasil se destaca entre todos los países de la muestra por ser el país latinoamericano que más empresas los asegura. Del total de 1.021 observaciones, un total de 448 son empresas brasileñas, lo que supone un $43,9 \%$ del total. El país que ocupa el segundo lugar por importancia en el aseguramiento es México (allí lo aseguran 51 empresas), si bien no es el segundo país que presentan informes de RSC sino el tercero. A continuación les sigue Chile con 32 empresas con aseguramiento, que de hecho, es el segundo país por presentación de informes de RSC. En realidad, tal como muestra la tabla 3, los casos de Brasil, México y Chile eran esperados por su mayor potencial económico con respecto a los demás, en términos de Producto Interior Bruto (PIB) a va- lores de Paridad de Poder Adquisitivo (PPA) y mayor número de grandes empresas a nivel mundial.

Los resultados de la tabla 3 sitúan a Brasil a la cabeza de Latinoamérica como pionera en la búsqueda de mayor credibilidad y transparencia en la información social y medioambiental que publican las empresas por medio de sus informes de RSC, seguida a gran distancia por Chile y México. Por otra parte, los países que no los aseguran son básicamente Uruguay, Bolivia y Honduras. Desde el punto de vista del aseguramiento centrándonos en 2010, hemos de destacar a Brasil, que sigue en primera posición y a México, que se sitúa en segundo lugar desplazando a Chile. Esto podría deberse a que en México existe el CEMEFI (Centro Mexicano para la filantropía), que promueve la actividad de RSC (Calderon, 2011).

\section{TABLA 3. Empresas que aseguran vs que no aseguran el informe de RSC por países}

\begin{tabular}{|c|c|c|c|c|c|c|c|c|}
\hline & & ASEGURAN & $\begin{array}{c}\text { NO } \\
\text { ASEGURAN }\end{array}$ & Total & Total (\%) & $\begin{array}{l}\text { *Posición } \\
\text { mundial en } \\
\text { PIB (PPA } \\
\text { 2011) }\end{array}$ & $\begin{array}{l}\text { *PIB (PPA) } \\
\text { Miles de } \\
\text { millones } \\
\quad \text { USD } \\
\quad 2011\end{array}$ & $\begin{array}{c}\text { **№ de empresas } \\
\text { de ese país en } \\
\text { Forbes Global } 2000 \\
2012\end{array}$ \\
\hline \multirow{9}{*}{ PAÍSES } & ARGENTINA & 13 & 67 & 80 & 7,84 & 21 & 709,7 & 0 \\
\hline & BRASIL & 122 & 326 & 448 & 43,88 & 7 & 2.284 & 33 \\
\hline & CHILE & 32 & 115 & 147 & 14,40 & 43 & 281 & 9 \\
\hline & COLOMBIA & 30 & 51 & 81 & 7,93 & 28 & 470.7 & 6 \\
\hline & COSTA RICA & 2 & 4 & 6 & 0,59 & 87 & 54,47 & 0 \\
\hline & MEXICO & 51 & 65 & 116 & 11,36 & 11 & 1.657 & 16 \\
\hline & PERÚ & 18 & 58 & 76 & 7,44 & 40 & 299,7 & 2 \\
\hline & URUGUAY & 0 & 9 & 9 & 0,88 & 88 & 52,02 & 0 \\
\hline & VENEZUELA & 4 & 1 & 5 & 0,49 & 34 & 368,8 & 1 \\
\hline \multicolumn{2}{|l|}{ Total } & 289 & 732 & 1.021 & 100 & & & \\
\hline
\end{tabular}

Fuente: elaboración propia y *The World Factbook y **Forbes.com

Después de haber analizado la muestra por año y por países, pasamos a estudiar las características empresariales de las empresas que someten su informe de RSC a aseguramiento.

Analizamos en primer lugar el tamaño y el sector a los que pertenecen las empresas que buscan mostrar una información de RSC de calidad por medio del aseguramiento. Así, en la tabla 4 podemos observar los detalles del porcentaje y de los valores absolutos de empresas pequeñas, medianas y grandes que aseguran versus que no aseguran sus informes de RSC. Como puede observarse, son las empresas pequeñas las que en menor medida se someten a aseguramiento externo $(4,69 \%)$ mientras que son las empresas grandes las que alcanzan un mayor número de empresas aseguradas $(32,33 \%)$. Nótese que aunque hay pocas empresas medianas en la muestra (61), de éstas, un alto porcentaje sí se aseguran (casi un 23\%). 
TABLA 4. Consideración de si aseguran vs no aseguran según el tamaño de la empresa

\begin{tabular}{l|c|c|c|c|c|c|c}
\hline & \multicolumn{3}{c}{ TAMAÑO DE LA EMPRESA } & \multicolumn{3}{c}{ GRANDES } \\
\cline { 2 - 8 } & \multicolumn{2}{|c|}{ PEQUENAS } & \multicolumn{2}{c}{ MEDIANAS } & 269 & $32,33 \%$ & 289 \\
\hline ASEGURAN & 6 & $4,69 \%$ & 14 & $22,95 \%$ & 563 & $67,67 \%$ & 732 \\
\hline NO ASEGURAN & 122 & 95,31 & 47 & $77,05 \%$ & 832 & $100 \%$ & 1.021 \\
\hline Total & 128 & $100 \%$ & 61 & $100 \%$ & & \\
\hline
\end{tabular}

Para establecer los sectores a los que pertenecen las empresas de la muestra, nos hemos basado en una clasificación en seis sectores: Petróleo y Energía, Materiales Básicos, Bienes de Consumo, Servicios de Consumo, Servicios Financieros, Tecnología y Telecomunicaciones y Otros. En la tabla 5 se muestran los distintos sectores en función de si la empresa asegura o no asegura.

\section{TABLA 5. Consideración de si aseguran vs no aseguran atendiendo al sector}

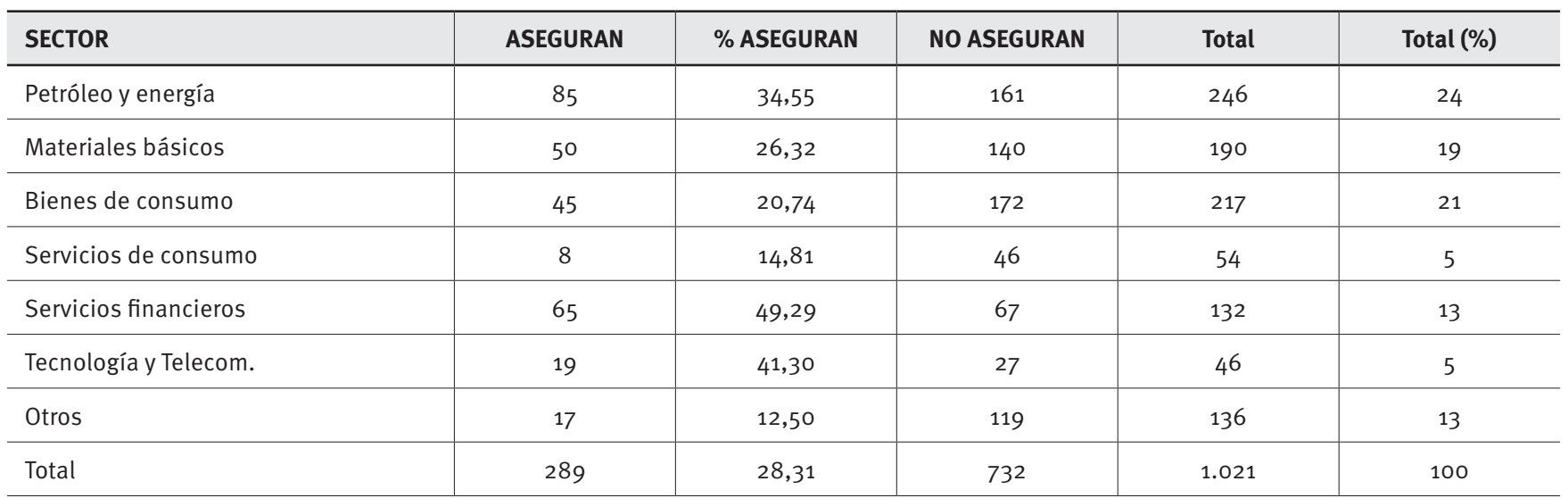

De las empresas aseguradas de nuestra muestra, el $24 \%$ corresponde al sector Petróleo y Energía, siendo el segundo, el de materiales básicos, con un 19\%. Hay que destacar que las empresas que más aseguran son las del sector Servicios Financieros con un $49,24 \%$ aunque le sigue muy de cerca el sector de Tecnología y Telecomunicaciones con más de un $41 \%$ y el del Petróleo y Energía (34,55\%). Al igual que nuestros resultados, la literatura señala que habitualmente son los sectores Petróleo y Energía y Servicios Financieros los que mayoritariamente se someten a aseguramiento (Simmett, Vanstraelen \& Chua, 2009; García-Benau, Zorio, \& Morente García, 2011; García-Benau, Sierra, \& Zorio, 2012).

Al haber una propensión tan dispar entre sectores hacia el aseguramiento de los informes de RSC, hemos reconvertido la variable sector en una variable dummy denominada "sector sensible". Esta variable toma el valor 1 en el caso de que se trate de un sector en que la proporción de las empresas que aseguran sus informes sea superior al $30 \%$ y o en otro caso -véase la tabla 5. Así, sector sensible incluye a los sectores de petróleo y energía, servicios financieros y tecnología y telecomunicaciones. La propensión de estos sectores a tener políticas de información sobre su actitud corporativa socialmente responsable se ratifica también en la literatura (Simnett, Vanstraelen, \& Chua , 2009, Kolk \& Perego, 2010). Del total de empresas, el $41,53 \%$ pertenecen al sector sensible y de estas casi un $40 \%$ se someten a aseguramiento. De aquellas que no pertenecen al sector sensible $(58,47 \%)$ se aseguran solamente un poco más del $20 \%$ (ver tabla 6).

TABLA 6. Consideración de si aseguran vs no aseguran atendiendo al sector sensible

\begin{tabular}{l|l|l|l|l|l}
\hline & \multicolumn{2}{l}{ SECTOR SENSIBLE } & \multirow{2}{*}{ Total } \\
\cline { 2 - 5 } & SI & NO & \\
\hline ASEGURAN & 169 & $39,86 \%$ & 120 & $20,10 \%$ & 289 \\
\hline NO ASEGURAN & 255 & $60,14 \%$ & 477 & $79,90 \%$ & 732 \\
\hline Total & 424 & $100 \%$ & 597 & $100 \%$ & 1.021 \\
\hline
\end{tabular}


Ya hemos comentado que el GRI ha velado siempre por mejorar la calidad de los informes de RSC. Para esta mejora (véase Zorio, Sierra, \& García-Benau, 2012), desde el año 2006 se establece una calificación de sus informes más clara al crearse un marco de referencia para dichos informes $(A+, B+y C+s i$ se someten a aseguramiento o A, B y C, en otro caso). De esta forma, se consigue promover que las organizaciones sean más sostenibles, transparentes, ofreciéndose además mayor credibilidad respecto a sus impactos en el mundo (GRI, 2002).

En los gráficos 1 y 2 se muestran el nivel de aplicación tanto de empresas que aseguran como de empresas que no aseguran. En el gráfico 1 se observa que $64,1 \%$ presentan un nivel $A+$ siendo $28,37 \%$ con $B+y$ 7,61\% con $C+$. Además, de aquellas empresas que no verifican, un $18,03 \%$ presenta un nivel $A$, un $29,37 \%$ B e igual porcentaje de C (gráfico 2). También habría que destacar que un $23,22 \%$ no declara su nivel de aplicación, especialmente en el año 2006.

\section{Resultados del estudio exploratorio}

En primer lugar, hemos llevado a cabo un análisis para detectar si las variables año, tamaño, sector sensible y país resultan significativas sobre la decisión de asegurar el informe de RSC (Q1), siendo la hipótesis nula $\mathrm{H}_{1}$ que no existe relación.

La tabla 7 muestra la tabla de contingencia de las variables correspondientes a las empresas que elaboran el aseguramiento del informe de RSC, distinguiendo si la empresa asegura o no el mismo. Para aquellas variables donde son categóricas (más de 2 categorías) se presentan además otras estadísticas para dar mayor solidez a los datos. Si observamos la tabla 7 todas las variables están relacionadas significativamente con el aseguramiento, excepto el año. Por tanto, salvo para la dimensión temporal hemos de aceptar la hipótesis alternativa de que el tamaño, la pertenencia a sector sensible y el país son significativas para explicar si la empresa asegura el informe de RSC.

\section{TABLA 7. Tablas de contingencias de empresas que aseguran vs empresas que no aseguran}

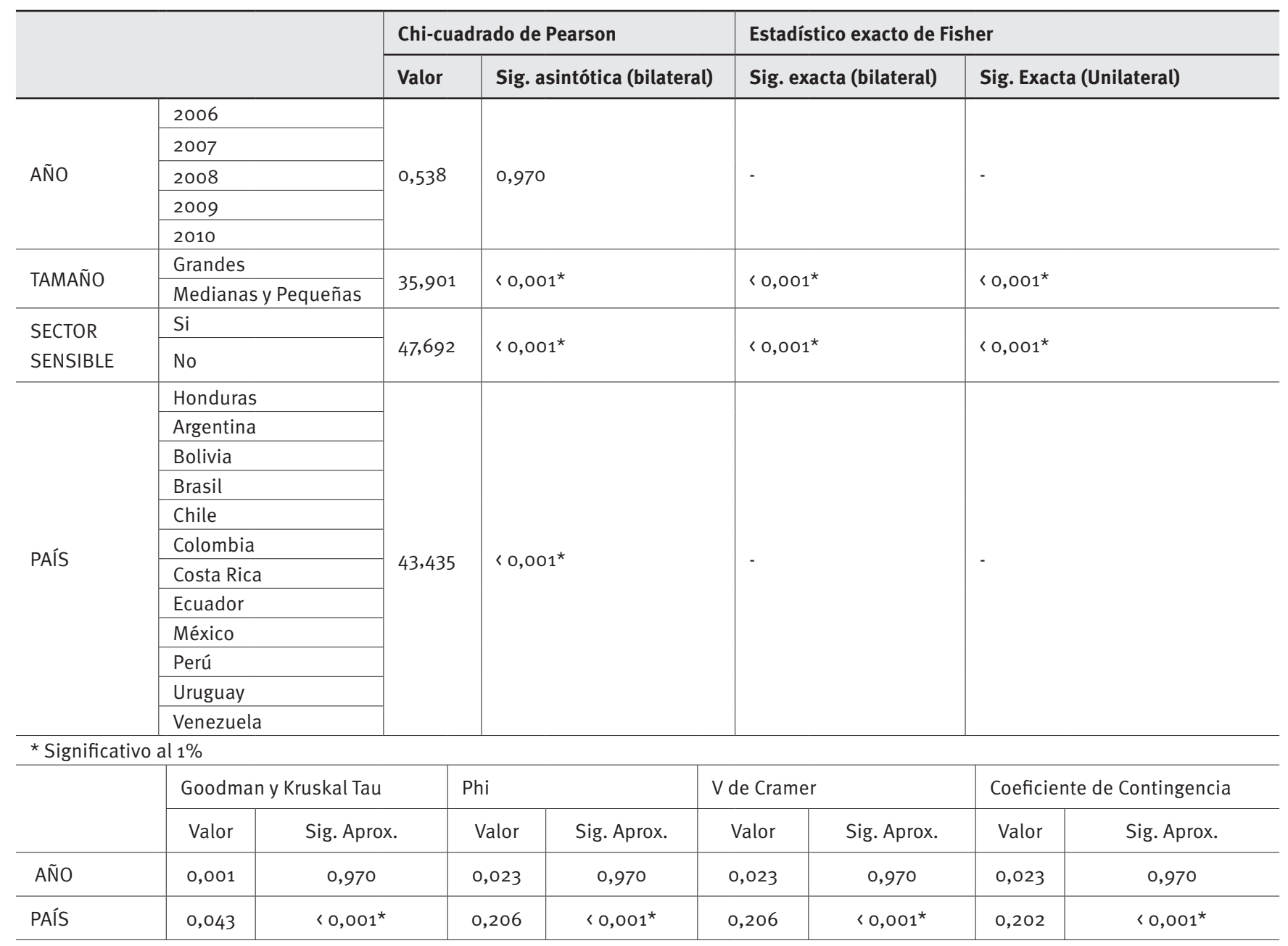

* Significativo al $1 \%$ 
Gráfico 1. Nivel de aplicación empresas aseguradas

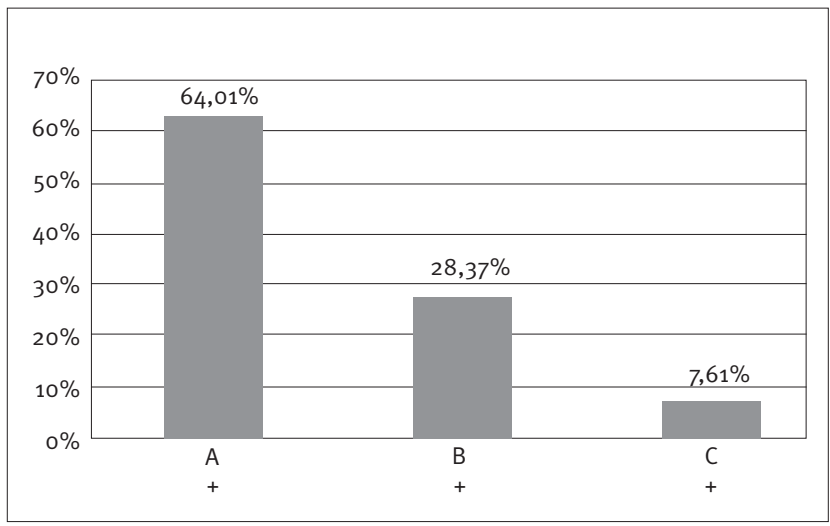

Gráfico 2. Nivel de aplicación empresas no aseguradas

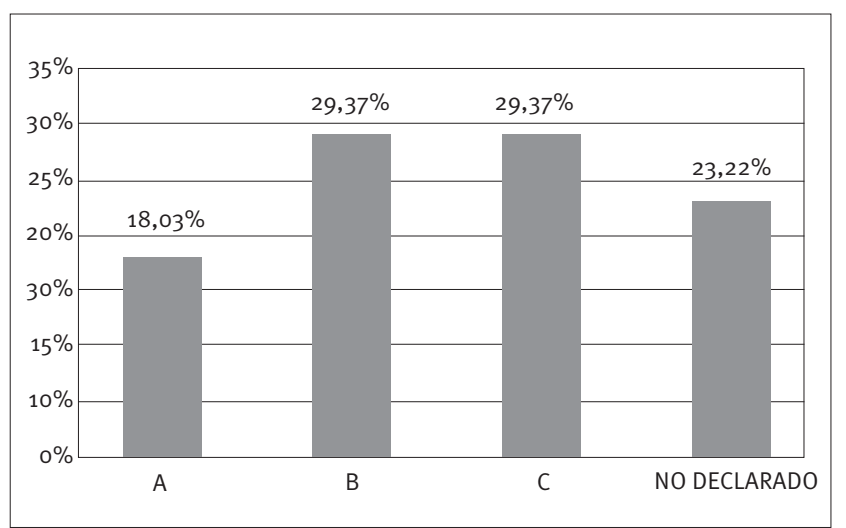

TABLA 8. Modelo de Regresión Logístico para predecir el aseguramiento del informe de RSC

\begin{tabular}{|c|c|c|c|c|c|c|c|}
\hline & & B & E.T. & Wald & gl & Sig. & $\operatorname{Exp}(B)$ \\
\hline \multicolumn{2}{|l|}{2007} & $-0,228$ & 0,323 & 0,497 & 1 & 0,481 & 0,796 \\
\hline \multicolumn{2}{|l|}{2008} & $-0,074$ & 0,304 & 0,060 & 1 & 0,807 & 0,928 \\
\hline \multicolumn{2}{|l|}{2009} & $-0,004$ & 0,291 & 0,000 & 1 & 0,989 & 0,996 \\
\hline \multicolumn{2}{|c|}{ TAMAÑO (Referencia: Medianas y pequeñas) } & 1,255 & 0,263 & 22,787 & 1 & $<0,001^{\star}$ & 3,507 \\
\hline \multicolumn{2}{|c|}{ SECTOR SENSIBLE (Referencia: No) } & 0,951 & 0,156 & 37,014 & 1 & $<0,001^{*}$ & 2,588 \\
\hline \multicolumn{2}{|c|}{ PAIS (Referencia: Honduras) } & & & 83,417 & 11 & $<0,001^{\star}$ & \\
\hline \multicolumn{2}{|l|}{ Chile } & $-2,522$ & 0,384 & 43,016 & 1 & $<0,001^{\star}$ & 0,080 \\
\hline \multicolumn{2}{|l|}{ Colombia } & $-2,124$ & 0,425 & 24,940 & 1 & $<0,001^{\star}$ & 0,120 \\
\hline \multicolumn{2}{|l|}{ Costa Rica } & $-2,370$ & 0,967 & 6,000 & 1 & $0,014^{\star \star}$ & 0,094 \\
\hline \multicolumn{2}{|l|}{ Ecuador } & $-2,133$ & 0,498 & 18,337 & 1 & $<0,001^{*}$ & 0,119 \\
\hline \multicolumn{2}{|l|}{ México } & $-1,417$ & 0,386 & 13,448 & 1 & $<0,001^{\star}$ & 0,242 \\
\hline \multicolumn{2}{|l|}{ Perú } & $-2,717$ & 0,445 & 37,338 & 1 & $<0,001^{*}$ & 0,066 \\
\hline \multicolumn{2}{|l|}{ Uruguay } & $-22,737$ & 12668,119 & 0,000 & 1 & 0,999 & 0,000 \\
\hline R2 Cox y Snell & \multicolumn{7}{|l|}{0,274} \\
\hline R2 Nagelkerke & \multicolumn{7}{|l|}{0,365} \\
\hline Clasificación Global & \multicolumn{7}{|l|}{$73,2 \%$} \\
\hline $\mathrm{N}$ & \multicolumn{7}{|l|}{1.021} \\
\hline
\end{tabular}


En la segunda parte de nuestro estudio exploratorio, por medio de un análisis de regresión logística (logit), vamos a identificar si el aseguramiento de las memorias de RSC depende de algunas variables corporativas, tales como el año, el tamaño y el sector sensible al que pertenece la empresa y la pertenencia a uno de los países de los que se componen la muestra (Q2), siendo la hipótesis nula $\mathrm{H} 2$ que no existe dicha dependencia.

En el modelo obtenido (ver tabla 8), el estadístico $\mathrm{Chi}^{2}$ es significativo con un valor de 326,431 ( $\rho$-valor: ,00o). Tanto los test de Cox y Snell y el de Nagelkerke muestran un buen ajuste del modelo, ya que no son significativos. De hecho, el porcentaje de clasificación correcta es de un 73,2\%.

En la tabla 8 podemos observar que la variable año no depende significativamente con el aseguramiento del informe de sostenibilidad. No obstante, hay que destacar que comparado con ser una empresa mediana o pequeña, el ser una empresa grande aumenta significativamente las probabilidades de tener un informe de sostenibilidad verificado (multiplica esta probabilidad por 3,507). Se observa que de la variable sector sensible depende significativamente si una empresa asegura o no su informe de sostenibilidad, es decir, comparado con no pertenecer a los sectores sensibles (petróleo y energía, servicios financieros y tecnología y telecomunicaciones), ser una empresa que sí pertenece a dichos sectores aumenta las probabilidades en 2,588 de asegurar el informe. Con respecto a la variable país, se evidencia que comparado con que la empresa sea de Honduras, resulta significativo ser del resto de países excepto Uruguay (esto es, multiplica por 0,038 la probabilidad si la empresa es de Argentina; 0,015 si es de Bolivia; 0,070 si es de Brasil; 0,080 si es de Chile; 0,120 si es de Colombia; 0,094 si es de Costa Rica; 0,119 si es de Ecuador; 0,242 si es de México; 0,066 si es de Perú; 0,457 si es de Venezuela).

En conclusión, hemos de rechazar la hipótesis $\mathrm{H}_{2}$ en términos de hipótesis nula y, en consecuencia, aceptar que la decisión de someter el informe de RSC a aseguramiento externo depende significativamente del tamaño de la empresa o de su pertenecía a un sector sensible o país en concreto.

\section{CONCLUSIONES}

La aparición dentro del panorama empresarial de otros grupos de interés, distintos a los accionistas, ha llevado a las empresas a asumir el reto de afrontar su responsabilidad social. La elaboración de informes de sostenibilidad o de Responsabilidad Social Corporativa les ha permitido, de una forma clara, mostrar a la sociedad su compromiso con los aspectos sociales y medioambientales.
La verificación de los informes de sostenibilidad dota a dichos informes de mayor credibilidad al ser realizada por un profesional calificado e independiente de la compañía. En este artículo hemos querido hacer nuestra aportación a la escasa literatura sobre informes de aseguramiento, centrándonos en estudiar el entorno de América Latina y realizando una aportación empírica que arroje alguna luz sobre las prácticas que están siguiendo las empresas.

Nuestra investigación abraca un periodo de estudio de cinco años, concretamente el período comprendido entre 2006 y 2010, ambos inclusive. El número de observaciones estudiadas ha sido de 1.021 procedentes de empresas de 12 países latinoamericanos.

Los resultados obtenidos son muy reveladores. De ellos se desprenden algunas conclusiones de gran interés sobre este campo, que enumeramos a continuación:

- Existe una clara tendencia alcista en cuanto a la presentación de informes de RSC, nótese que el número de empresas que presentan dicho informe se ha cuadruplicado en Latinoamérica en tan solo 5 años. El aseguramiento de los informes de RSC también ha crecido de igual modo en este período. En nuestra opinión, ello es debido al reciente desarrollo de este tipo de políticas de información empresarial y al auge que a nivel internacional están tomando organizaciones como el GRI.

- Brasil se sitúa en una posición líder, tanto en presentación de informes de RSC como en la realización de informes de aseguramiento. México y Chile le siguen en cuanto a la presentación de informes de RSC, y México se sitúa en segundo lugar en cuanto al aseguramiento. Ello es debido sin duda al mayor desarrollo económico de estos países (Brasil figura como $7^{\text {a }}$ potencia mundial en términos de PIB según los datos del FMI de 2012), en comparación con sus vecinos de Latinoamérica. Probablemente el mayor escrutinio internacional al que está sometido Brasil, tras postularse en 2004 como sede de la Copa Mundial de fútbol en 2014 y su posterior carrera para ser sede olímpica en 2016, ayudan en cierto modo a explicar la mayor transparencia de sus empresas en términos de sostenibilidad.

- Las empresas que más aseguran son las pertenecientes a los sectores Servicios Financieros, Tecnología y Telecomunicaciones, y Petróleo y Energía. Este resultado es coincidente con la literatura previa, que señala una mayor sensibilidad de este tipo de empresas por mostrar a todos sus stakeholders un comportamiento comprometido con el entorno. 
- Los resultados del modelo de regresión logística revelan que las variables corporativas que resultan significativas para la decisión de someter el informe de sostenibilidad a aseguramiento son el tamaño de la empresa, el país en el que se ubica y su pertenencia al denominado sector sensible.

\section{LIMITACIONES Y FUTURAS LÍNEAS DE INVESTIGACIÓN}

Para terminar, debemos reconocer que nuestro trabajo adolece de algunas limitaciones, como son la utilización de una muestra un tanto escasa para algunos países y una metodología eminentemente descriptiva. Ambos aspectos pueden justificarse en la medida en que, por un lado, se ha partido de toda la información disponible y pensamos que descartar algunos países empobrecería el estudio de la cuestión en toda la región. Por otro lado, consideramos que la metodología utilizada resulta adecuada, suficiente y sólida para nuestros objetivos y está en línea con la literatura disponible en este campo concreto. Nótese que se trata de un ámbito de estudio muy novedoso y la atención de este trabajo se centra en estudiar el fenómeno del aseguramiento en Latinoamérica. No obstante, en posteriores trabajos será desde luego muy interesante plantear innovaciones metodológicas en este terreno, lo que abre nuevas vías a la investigación futura. Asimismo, este trabajo puede sentar las bases para la comparación futura con otras áreas geográficas o el estudio comparado de las técnicas de aseguramiento por equipos interdisciplinarios entre las distintas firmas de auditoría y consultoría que acometen estos encargos, entre otras cuestiones de máxima actualidad.

Se trata sin duda de un tema muy relevante y actual para el conocimiento sobre administración de empresas, debido a la apuesta decidida de las empresas para invertir en RSC y en su aseguramiento, incluso en tiempos de crisis económica (véase García-Benau, Sierra, \& Zorio, 2013). Un tratamiento administrativo correcto y transparente de las acciones comprometidas con el entorno por parte de las organizaciones, las pone en el camino de la evaluación de los programas. De esta forma, la contribución más importante de este trabajo es centrar la atención sobre las recomendaciones internacionales en este campo y más concretamente sobre el análisis de las prácticas de aseguramiento en Latinoamérica.

No podemos finalizar nuestro artículo sin subrayar que los resultados obtenidos son muy alentadores. Ponen de manifiesto una clara tendencia en las empresas de América Latina que alcanzará probablemente cuotas muy importantes en los próximos años. Ello es especialmente interesante si tenemos en cuenta que la tendencia internacional está llevando a que las empresas lleven a cabo cambios en su política de comunicación y avancen en lo que viene denominándose la elaboración del Informe Integrado que sintetiza de forma clara y precisa la información tanto financiera como no financiera de las corporaciones.

\section{REFERENCIAS}

Accountability. AA1000AS Assurance Standard, (2003). Disponible en: www.accountability21.com.Accountability. AA1000AS Assurance Standard. (2008). Disponible en: www.accountability21.com.

Adams, C. A, \& Evans, R. (2004). Accountability, completeness, credibility and the audit expectations gag. Journal of Corporate Citizenship, 14, 97-115, 2004.

Aragón-Correa, J. A, Hurtado-Torres, N, Sharma, S, \& García-Morales, V. J. (2008). Environmental strategy and performance in small firms: a resource-based perspective. Journal of Environmental Management, 86 (1), 88-103.

Arroyo, J. (2012). Promover al promotor: el estado ante la responsabilidad social empresarial. CENTRUM Católica's working paper series Disponible en: http://centrum.pucp.edu.pe/pdf/working_paper_series/CERES_ WP2012-09-0004.pdf.

Asif, M, Searcy, C, Dos Santos, P, \& Kensah, D. (2012). A review of Dutch corporate sustainable development reports. Corporate Social Responsibility and Environmental Management, DOI: 10.1002/csr.1284.

Austin, J. E, Gutiérrez, R, Ogliastri, E, \& Reficco, E. A. (2009). Aprovechar la convergencia. Academia, Revista Latinoamericana de Administración, 43, 93-106.

Berbel Giménez, G, Reyes Gómez, J. D, \& Gómez Villegas, M. (2007). La responsabilidad social en las organizaciones (RSO): análisis y comparación entre guías y normas de gestión e información. Innovar, 17 (29), 27-48.

Calderon, M. (2011). CSR in Latin America and South East Asia analysis of the corporate communication of top local companies. International Research Journal of Finance and Economics, 73, 56-74.

Carroll, A. B. (1999). Corporate social responsibility: evolution of a definitional construct. Business and Society, 38 (3), 268-295.

Fédération des Experts Comptables Européens (FEE). (2006). Key issues in sustainability assurance: an overview. Bruselas: Fédération des Experts Comptables Européens.

Feldman, P. M, \& Reficco, E. (2012). Impacto de la responsabilidad social empresarial en comportamiento de compra y disposición a pagar de consumidores bogotanos. CENTRUM Católica's Working Paper. Disponible en: http://scholar.googleusercontent.com/scholar?q=cache:wellnn23wlkJ:scholar.google.com/+reficco+rsc\&hl=es\&as_sdt=0,5.

Fernandez Orellano, V. I, \& Quiota, S. (2011). Análise do retorno dos investimentos socioambientais das empresas brasileiras. RAE- Revista de Administração de Empresas, 51 (5), 471-484.

Fifka, M. S. (2013). Corporate responsibility reporting and its determinants in comparative perspective - a review of the empirical literature and a meta-analysis. Business Strategy and the Environment, 22 (1), 1-35. 
Fonseca, A. (2010). How credible are mining corporations' sustainability reports? critical analysis of external assurance under the requirements of the international council on mining and metals. Corporate Social Responsibility and Environmental Management, 17, 355-370.

García-Benau, M. A, Zorio, A, \& Morente García. J. (2011). El aseguramiento de los informes de sostenibilidad: normativa internacional y tendencias en España. 8th Spanish Conference on Social and Environmental Accounting Research: Burgos.

García-Benau, M. A, Sierra, L, \& Zorio, A. (2012). La verificación de la memoria de sostenibilidad en un contexto europeo. Globalización, Competitividad y Gobernabilidad, 6 (2), 66-80.

García-Benau, M. A, Sierra, L, \& Zorio, A. (2013). Financial crisis impact on sustainability reporting. Management Decision, 51 (7), 1-21.

Giannarakis, G, \& Theotokas, I. (2011). The effect of financial crisis in corporate social responsibility performance. International Journal of Marketing Studies, 3 (1), 213-234.

Global Reporting Initiative (GRI). (2002). G3 Sustainability Reporting Guidelines, Amsterdam: Global reporting Initiative.

Gómez Villegas, M. (2008). Evaluación crítica de un caso de RSE en una empresa de cemento en Colombia. In: Carlos Eduardo Martínez Fajardo, Responsabilidad social empresarial: una reflexión desde la gestión, lo social y ambiental (Cap. 6). Bogotá: Colombia: Editora: Centro Editorial FCE - CID.

Gray, R. H; Milne, M. J. (2004). Towards reporting on the triple bottom line: mirages, methods and myths. In: A Henriques and J. Richardson (Eds), The Triple Bottom Line: Does it All Add Up?, (Chapter 7, pp. 70-80). Earthscan: London.

Hasan, M, Maijoor, S, Mock, T. J, Roebuck, P, Simnett, R, \& Vanstraelen, A. (2005). The different types of assurance services and levels of assurance provided. International Journal of Auditing, 9, 91-102.

International Accounting Auditing Standard Board (IAASB). (2003). International Standard on assurance engagements 3000-Assurance engagements other than audits or reviews of historical information, New York: IFAC.

Kang, K. H, Lee, S, \& Huh, C. (2010). Impacts of positive and negative corporate social responsibility activities on company performance in the hospitality industry. International Journal of Hospitality Management, 29 (1), 72-82.

Knechel, W. R, Wallage, P, Eilifsen, A, \& Praag, B. (2006). The demand attributes of assurance services providers and the role of independent accountants. International Journal of Accounting, 10 (2), 143-162.

Kolk, A. (2008). Sustainability, accountability and corporate governance: exploring multinationals' reporting practices. Business Strategy and the Environment, 17 (1), 1-15.
Kolk, A, \& Perego, P. (2010). Determinants of the adoption of sustainability assurance statements: an international investigation. Business Strategy and the Environment, 19 (3), 182-198.

Kpmg. KPMG International Survey of Corporate Responsibility Reporting (2011). Disponible en: http://www.kpmg.com/global/en/ issuesandinsights/articlespublications/corporate-responsibility/ pages/2011-survey.aspx.

Moroney, R, Windsor, C, \& AW, Y. T. (2012). Evidence of assurance enhancing the quality of voluntary environmental disclosures: an empirical analysis. Accounting \& Finance, 52 (3), $903-939$.

Nelling, E, \& Webb, E. (2009). Corporate social responsibility and financial performance: the "virtuous circle" revisited. Review Quantitative Finance and Accounting, 32 (2), 197-209.

O'dwyer, B. (2011). The case of sustainability assurance: constructing a new assurance service. Contemporary Accounting Research, 28 (4), 1230-1266.

Orlitzky M, Schmidt F. L, \& Rynes SL. (2003).Corporate social and financial performance: a meta-analysis. Organizations Studies, 24 (3), 403-441.

Porter, M. E, \& Kramer, M. R. (2002). The competitive advantage of corporate philanthropy. Harvard Business Review, 80 (12), 5-16.

Reficco, E. (2009). Menos voluntarismo, más innovación: negocios inclusivos en Latinoamérica. INCAE Business Review, 1 (9), 38-48.

Reficco, E. (2010). Negocios inclusivos y responsabilidad social: un matrimonio complejo. Debates IESA, 15 (3), 14-17.

Reficco, E \& Ogliastri, E. (2009). Empresa y sociedad en América Latina: una introducción. Academia. Revista Latinoamericana de Administración, 43, 1-25.

Simnett, R, Vanstraelen, A, \& Chua, W. F. (2009). Assurance on sustainability reports: an international comparison. The Accounting Review, 84 (3), 937- 967.

Simpson, W. G, \& Kohers, T. (2002). The link between corporate social and financial performance: evidence from the banking industry. Journal of Business Ethics, 35 (2), 97-109.

Unión Europea, UE. (2010). Informes de sostenibilidad en la Unión Europea. Fondo de Reputación Corporativa: Bruselas.

Wright, P, \& Ferris, S. P. (1997). Agency conflict and corporate strategy: the effect of divestment on corporate value. Strategic Management Journal, 18 (1), 77-83.

Zorio, A, Sierra, L, \& García-Benau, M. A. (2012). Aseguramiento del informe de sostenibilidad en España y Latinoamérica, Cádiz: Encuentro de profesores de contabilidad (ASEPUC), 2012.

Zorio, A., García-Benau, M. A. and Sierra, L. (2013), Sustainability Development and the Quality of Assurance Reports: Empirical Evidence. Bus. Strat. Env., 22: 484-500. doi: 10.1002/bse.1764 